\title{
Algorithm of the Radar Imaging by Using the Wideband Signals with the Distorted Signal Phases
}

\author{
Yulia V. Zhulina \\ Interstate Corporation "Vympel”, 125319 Moscow, Russia \\ Email:yulia_julina@mtu-net.ru \\ Received 16 December 2002 and in revised form 30 June 2003
}

\begin{abstract}
The problem of restoring an image by its Fourier transform is considered when the Fourier transform contains phase distortions. The nature of these distortions and their values are arbitrary. The criterion for the quality of the phase distortion estimates is suggested. It can be used to select the image which is mostly like the true one. The nature of the true image is also arbitrary. The only condition for the true image is that it is real and positive for all the points of the restored area. The other condition for the task is that the recovered image is calculated as the absolute value of the inverse Fourier transform. The algorithm for the search of the compensating phases satisfying the criterion is not considered for the general case; however, the task of the radar imaging based on the wideband signal and the time synthesis of the aperture is treated in detail. The physical basis for the task is a wideband pulse radar signal reflected by a moving object. As a result, a two-dimensional aperture is synthesized along the range, due to the super resolution, and along the velocity, according to the motion of the object. The radar signals are received by a single receiver. The image is reconstructed on the basis of these signals by using the maximum likelihood technique. The method uses the coherent processing of the signals. In practice, the coherence can be destroyed (due to some atmospheric turbulence or equipment instability, due to some inaccuracy in defining the motion). We assume that the objects to be observed are located at the far zone. For this task and on the basis of the suggested criterion, we develop an approximate algorithm for searching the best compensating phases in the radar signal. The quality of the images is tested with the help of simulation.
\end{abstract}

Keywords and phrases: Fourier transform, phase distortions, radar imaging.

\section{INTRODUCTION}

A lot of tasks in optics, tomography, radar, and astronomy are connected with image recovery by using the Fourier spectra. The synthesis of the procedures for performing image filtering, enhancement, restoration, and segmentation presupposes similar tasks. These spectrum data include some amplitude and (or) phase distortions of various types very often. The problem of compensating these distortions with the aim of achieving high quality of image restoration is actual, and different techniques were suggested to solve this problem both in radar science $[1,2,3,4,5,6,7,8,9,10,11]$ and in optics $[12,13,14,15,16,17,18,19,20,21,22]$. It is important to notice that in the Fourier optics, one of the most topical tasks is the problem of simultaneous restoration of the optical response function and of the object image in the presence of some phase distortions related to atmospheric turbulences. In the latter case, the restoration task is a problem of blind deconvolution, but not that of the Fourier spectrum restoration. Nevertheless, in the tasks of coherent optics, such as laser optics, the problem of the Fourier spectrum restoration may be quite topical.
As for the radar imaging, the works which are most close to our subject consider the synthetic aperture autofocus methods. One approach is to choose a range cell, containing a bright scatterer [8]. For a complex target, that does not have a bright scatterer, the estimation of the pulse-to-pulse phase difference of the reference point can be calculated by taking the phase differences for each range cell and averaging them with the weights proportional to the amplitudes of the signal in each of the range cells [2]. The alternative approach suggests selecting only the range cells with strong scatterers $[3,4,9]$. Another method, based on the image contrast, has also been proposed for synthetic aperture autofocusing $[5,6]$. In $[1]$, the estimation of the complex phase vector was formed by the exponential function of the phase rather than the phase itself. In [7], the eigendecomposition of the signal vector covariance matrix is used for correcting some aperture phase errors.

During the recent 5-7 years, a powerful theory of constructing mathematical objects (signals, functions, and images) in a vector space, satisfying some multiple constraints, was developed. It is named "vector space projection method" [23]. All the constraint spaces are convex and have 
a nonempty space of intersection. The basic idea of the solution is as follows: an initial vector (i.e., an initial object) is being projected to each of the sets of constraints step by step. The unitary projecting to the full set of restrictions completes the first iteration. The vector, which is obtained after the iteration, is considered to be the initial one for the next iteration. The iterative process converges to the vector, satisfying all the constraints simultaneously. This theory was successfully applied to the filter design tasks with the classical constraints: passband fluctuations, stopband attenuation, transition band behavior, and filter length (length of the impulse response) $[24,25]$. In [26], the task of synthesis, a desirable form of the far-field radiation pattern, is resolved. A task of a self-healing in the arrays antennas is also considered.

The presented paper considers the case when there are no amplitude distortions, but the phase distortions are presented in the Fourier transform. The nature of these distortions and their values are arbitrary. The criterion for the quality of estimating the phase distortions is suggested and it can be used to select the image mostly close to the true one. The nature of the true image is also arbitrary. The condition for the task is that the recovered image is calculated as the absolute value of the inverse Fourier transform. The algorithm for the search of the compensating phases satisfying the criterion is considered for the task of the radar imaging by using the wideband signal and the time synthesis of the aperture. For this task on the basis of the suggested criterion, we will develop an approximate algorithm for finding the best compensating phases in the radar signal. The quality of the images is tested with the help of simulation.

\section{CRITERION FOR FINDING THE BEST IMAGE OBTAINED AS A RESULT OF THE INVERSE FOURIER TRANSFORM}

We assume that the object to be reconstructed is placed in the far Fraunhofer zone, so that the received field is a plane space wave and the image $\hat{E}(\vec{s})$ is being constructed as the inverse two-dimensional Fourier transform of the received field $\hat{F}(\vec{R})$,

$$
\hat{E}(\vec{s})=\frac{1}{(2 \pi)^{2}} \int_{\Omega_{\vec{R}}} \hat{F}(\vec{R}) \exp \left(-j k_{0} \vec{R} \vec{s}\right) d^{2} \vec{R}
$$

Here, $k_{0}=2 \pi / \Lambda \rho$, where $\Lambda$ is the wavelength of the signal, $\rho$ is the range, $\Omega_{\vec{R}}$ is an area of the receiving aperture, $\vec{s}$ is a coordinate vector in the object space, and $\vec{R}$ is a vector in the Fourier space.

Suppose that the received field $\hat{F}(\vec{R})$ is distorted and is connected with the true field $F(\vec{R})$ by the relationship

$$
\hat{F}(\vec{R})=\exp (j \theta(\vec{R})) F(\vec{R}) .
$$

Here, $\theta(\vec{R})$ is the function of the phase distortions. In this case, the inverse Fourier transform (1) produces a distorted image.
We can take any arbitrary estimation of the phase distortions $\hat{\theta}(\vec{R})$ and include this estimate as a compensating multiplier in the expression (1). As a result, we get the next expression for $\hat{E}(\vec{s})$ instead of (1):

$$
\hat{E}(\vec{s})=\frac{1}{(2 \pi)^{2}} \int_{\Omega_{\vec{R}}} \hat{F}(\vec{R}) \exp \left(-j k_{0} \vec{R} \vec{s}\right) \exp (-j \hat{\theta}(\vec{R})) d^{2} \vec{R} .
$$

The function $\hat{\theta}(\vec{R})$ is unknown and must be found.

Further, we will designate the true image $E(\vec{s})$ and will limit the considerations to the images $E(\vec{s})$, for which

$$
E(\vec{s})=A(\vec{s}) \exp \left(j \varphi_{0}\right), \quad \varphi_{0}=\text { const. }
$$

According to (3), we can determine the estimate of the modulus $\hat{A}(\vec{s})$ :

$$
\begin{aligned}
\hat{A}(\vec{s}) & =|\hat{E}(\vec{s})| \\
& =\frac{1}{(2 \pi)^{2}}\left|\int_{\Omega_{\vec{R}}} \hat{F}(\vec{R}) \exp \left(-j k_{0} \vec{R} \vec{s}\right) \exp (-j \hat{\theta}(\vec{R})) d^{2} \vec{R}\right| .
\end{aligned}
$$

Suppose that we can do a complete exhaustive search among the possible estimates of phase distortions $\hat{\theta}(\vec{R})$ and we have no prior knowledge about the true function

$$
A(\vec{s})=|E(\vec{s})|
$$

so, for every new function $\hat{\theta}(\vec{R})$, we get a new function $\hat{A}(\vec{s})$ according to (5). Which of these functions $\hat{A}(\vec{s})$ in the process of the search should be chosen as the best one?

In Appendix A, we show that in this case the best function $\hat{A}_{\text {best }}(\vec{s})$ among all possible $\hat{A}(\vec{s})$ satisfies the condition

$$
\int_{\Omega_{\vec{s}}} \hat{A}_{\text {best }}(\vec{s}) d^{2} \vec{s} \leq \int_{\Omega_{\vec{s}}} \hat{A}(\vec{s}) d^{2} \vec{s}
$$

where $\Omega_{\vec{s}}$ is the area, a priori occupied by the object.

So, the best estimation of the image must provide the minimum to the integral over the area of restoration $\Omega_{\vec{s}}$ compared to all other estimates.

The first impression that the trivial result $\hat{A}_{\text {best }}(\vec{s}) \equiv 0$ is the solution to the task is a mistake, since, according to (2) and (5), the spectrum of $\hat{E}_{\text {best }}(\vec{s})$ is equal to

$$
\hat{F}_{\text {best }}(\vec{R})=\exp \left(j\left(\theta(\vec{R})-\hat{\theta}_{\text {best }}(\vec{R})\right)\right) F(\vec{R}),
$$

and Parseval's theorem gives

$$
\begin{aligned}
\int_{\Omega_{\vec{s}}} \hat{A}_{\text {best }}^{2}(\vec{s}) d^{2} \vec{s} & =\int_{\Omega_{\vec{s}}}\left|\hat{E}_{\text {best }}(\vec{s})\right|^{2} d^{2} \vec{s} \\
& =\frac{1}{(2 \pi)^{2}} \int_{\Omega_{R}}|F(\vec{R})|^{2} d^{2} \vec{R} \\
& =\int_{\Omega_{\vec{s}}} A^{2}(\vec{s}) d^{2} \vec{s} .
\end{aligned}
$$


So, the full energy of the image remains constant in the process of exhaustive search of phases $\hat{\theta}(\vec{R})$.

\section{THE TASK OF THE RADAR IMAGING WITH THE DISTORTED SIGNAL PHASES}

We consider a single radar transmitter, which radiates the pulse signals $u_{n}(t)(n=1, \ldots, N)$ at the moments $t_{1}, t_{2}, \ldots, t_{N}$ in the direction of the object under observation, where $N$ is the number of emitted pulses and $u_{n}(t)$ is the shape of a complex pulse signal, emitted by the transmitter.

For example, the shape $u_{n}(t)$ can be written as

$$
\begin{aligned}
u_{n}(t)= & u\left(t-t_{n}\right) \\
= & \exp \left(-\alpha \cdot\left(t-t_{n}\right)^{2}\right. \\
& \left.\quad+j\left(\omega\left(t-t_{n}\right)+\Delta \omega \cdot\left(t-t_{n}\right)^{2}\right)\right) .
\end{aligned}
$$

Formula (10) corresponds to a Gaussian pulse with the carrier frequency $\omega$ and the linear frequency modulation.

The shape (10) is not necessary; the basic condition is that the pulse $u_{n}(t)$ should have a wideband of frequency modulation.

Let $\vec{R}_{\mathrm{obj}}\left(t_{n}\right)$ be the position of the center of object's masses at time $t_{n}$ and $\vec{R}_{0}$ is the location of the radar receiver in the coordinate frame with the origin in the center of the Earth.

For each moment $t_{n}$, we have $\vec{e}_{n}$ which is the normalized vector of viewing the object from the receiver:

$$
\vec{e}_{n}=A_{\mathrm{obj}}^{-1}\left(t_{n}\right) \frac{\vec{R}_{\mathrm{obj}}\left(t_{n}\right)-\vec{R}_{0}}{\left|\vec{R}_{\mathrm{obj}}\left(t_{n}\right)-\vec{R}_{0}\right|},
$$

where $A_{\text {obj }}\left(t_{n}\right)$ is the matrix of object's rotation around its center of masses and $A_{\mathrm{obj}}^{-1}\left(t_{n}\right)$ is the inverse matrix.

We will not consider the problems of estimating the rotation parameters and tracking objects here. We assume that they have already been solved accurately enough in $[1,2,3,4,5,6,27]$. We assume, as well, that the orbital motion of the object produces the synthesis of the aperture in time. Thus, we assume that $A_{\mathrm{obj}}\left(t_{n}\right)$ is the unit matrix.

The complex video signal $Z_{n}(\lambda)(n=1, \ldots, N)$ with the pulse number $n$ at the output of the matched filter of the radar receiver can be presented as, (in the far zone) $[2,28$, $29,30]$,

$$
\begin{aligned}
Z_{n}(\lambda)= & \int_{S_{\mathrm{LIGHT}}} E^{*}(\vec{s}) \exp \left(j \omega 2 \vec{e}_{n}^{T} \cdot \frac{\vec{s}}{c}\right) \\
& \cdot C_{0}\left(\lambda-2 \vec{e}_{n}^{T} \cdot \frac{\vec{s}}{c}\right) d^{2} \vec{s} \exp \left(-j \theta_{n}\right)+m_{n}(\lambda) \\
= & \bar{Z}_{n}(\lambda)+m_{n}(\lambda) .
\end{aligned}
$$

Here, $S_{\text {LIGHT }}$ is the area of the object surface irradiated by transmitter, $C_{0}(\lambda)$ is the autocorrelation function of the radar pulse (the expression for it is given below), $\vec{s}$ is the coordinate vector of some element of object's surface, $d^{2} \vec{s}$ is the element of the surface in the coordinate frame connected with the object center of masses, $E(\vec{s})$ is the complex-reflected signal of the scatterer located on the surface of the rough object in the point $\vec{s}, \lambda$ is a range (in time representation) with respect to the center mass (after the compensation of the motion of the mass center), $m_{n}(\lambda)$ is the additive complex Gaussian noise at the output of the filter as in the function of time $\lambda$ with power $\sigma_{\text {noise }}^{2}$ and zero average and $\vec{e}_{n}^{T}$ is the transposed vector of observation. In (12), $\theta_{n}$ is the phase distortion, generated by the atmosphere on the way receiver-object and back.

Besides, the phase distortion may be caused by the phase instability in the receiver. Some additional source of phase distortions is the uncertainty of the motion of the object mass center evaluation. The motion of the center of masses gives shifts both to the position of each signal and to its phase. If the phase shift, caused by this motion, is not accurately enough compensated [1], this will lead to some phase distortions of signals.

Further in (12), $c$ is the velocity of light, $\omega$ is the carrier frequency of the radar signal, and $N$ is the number of pulses used in processing. Each signal $\bar{Z}_{n}(\lambda)$ is the middle value of video signal.

The autocorrelation function $C_{0}(\lambda)$ in (12) can be expressed through the form of pulse $u(t)$ as follows:

$$
C_{0}(\lambda)=\int_{-\infty}^{\infty} u^{*}(t) u(t-\lambda) d t
$$

$E(\vec{s})$ in $(12)$ is unknown.

We will designate the modulus of the function

$$
A(\vec{s})=|E(\vec{s})|
$$

as the unknown radar image of the object. It should be constructed by using the signals $Z_{n}(\lambda)(n=1, \ldots, N)$.

The form of the receiver diagram is not included in (12), because we assume that it is wider than the dimensions of the object, and the tracking of the object is accurately enough performed.

This task is a classical one in the radar tracking theory and practice $[10,11,31,32,33,34]$. The most significant results of the radar imaging practice by using wideband signals are presented in $[32,35,36,37,38]$. Here, we are developing the approach to the restoration of the coherence in the radar signals investigated earlier in $[10,11]$.

\section{MAXIMUM LIKELIHOOD ALGORITHM}

Assuming that the noise $m_{n}(\lambda)$ is statistically independent of different numbers $n$, we can write the logarithm of the likelihood function for all the signals $Z_{n}(\lambda)(n=1, \ldots, N)$ :

$$
\operatorname{Ln} P=-\frac{1}{\sigma_{\text {noise }}^{2}} \sum_{n=1}^{N} \int_{\lambda_{\min }}^{\lambda_{\max }}\left|Z_{n}(\lambda)-\bar{Z}_{n}(\lambda)\right|^{2} d \lambda .
$$

Here, $\lambda_{\min }$ is the minimum value of $\lambda$ among the arguments 
of all the signals $Z_{n}(\lambda)(n=1, \ldots, N)$, and $\lambda_{\max }$ is the maximum value of $\lambda$ among the arguments of all the signals $Z_{n}(\lambda)$ $(n=1, \ldots, N)$.

The maximum likelihood estimate of $E(\vec{s})$ can be obtained from the equations

$$
\frac{\delta \operatorname{Ln} P}{\delta \operatorname{Re} E(\vec{s})}=0, \quad \frac{\delta \operatorname{Ln} P}{\delta \operatorname{Im} E(\vec{s})}=0 .
$$

These equations are the functional derivatives of the functional $\operatorname{LnP}$ with respect to the functions $\operatorname{Re} E(\vec{s})$ and $\operatorname{Im} E(\vec{s})$.

Appendix B proves that the solution of (16) gives the estimation of $\hat{E}(\vec{s})$ (to a constant factor):

$$
\hat{E}(\vec{s})=\frac{1}{N} \sum_{n=1}^{N} Z_{n}^{*}\left(\tau_{n}(\vec{s})\right) \exp \left(-j\left(\hat{\theta}_{n}-\omega \tau_{n}(\vec{s})\right)\right) .
$$

Here, $\hat{\theta}_{n}$ is the estimation of the phase distortions while

$$
\tau_{n}(\vec{s})=2 \vec{e}_{n}^{T} \cdot \frac{\vec{s}}{c}, \quad(n=1, \ldots, N)
$$

is the time delay of the number $n$ signal from the surface point of the object corresponding to $\vec{s}$.

To determine the coordinate frame of the image $\hat{E}(\vec{s})$, we will introduce the axes of the coordinate frame $\vec{s}$. The $Y$-axis is directed as the average line of sight (and upwards in all the pictures). In other words, the direction of the $Y$-axis coincides with the direction $\vec{e}_{N / 2}$. So,

$$
\vec{e}_{\vec{s}, Y}=\vec{e}_{N / 2}
$$

The $X$-axis is directed as the projection of the average (over all the pulses) object velocity vector to the plane, orthogonal to the vector $\vec{e}_{N / 2}$ :

$$
\vec{e}_{\vec{s}, X}=\frac{\vec{v}_{N / 2}-\vec{e}_{N / 2}\left(\vec{e}_{N / 2} \cdot \vec{v}_{N / 2}\right)}{\left|\vec{v}_{N / 2}-\vec{e}_{N / 2}\left(\vec{e}_{N / 2} \cdot \vec{v}_{N / 2}\right)\right|} .
$$

Here, $\vec{v}_{N / 2}$ is the vector of the object velocity in the coordinate frame, connected with the center of the earth, in which the vector $\vec{e}_{N / 2}$ is determined. The $Z$-axis is orthogonal to the axes $X$ and $Y$. The vector $\vec{e}_{\vec{s}, Z}$ completes the vectors $\vec{e}_{\vec{s}, X}$ and $\vec{e}_{\vec{s}, Y}$ to the right-hand coordinate frame.

It should be noted that in the direction $\vec{e}_{\vec{s}, Z}$, the resolution of the image recovery system is zero. Therefore, the threedimensional image $\hat{E}(\vec{s})$ is ,in fact, a two-dimensional image. We will assume everywhere that

$$
\hat{E}(\vec{s})=\hat{E}\left(\vec{s}_{X}, \vec{s}_{Y}, 0\right) .
$$

If (17) is calculated, the image estimation $\hat{A}(\vec{s})$ is obtained by using the formula

$$
\hat{A}(\vec{s})=|\hat{E}(\vec{s})|
$$

and, according to (21),

$$
\hat{A}(\vec{s})=\left|\hat{E}\left(\vec{s}_{X}, \vec{s}_{Y}, 0\right)\right| .
$$

Algorithm (17) can work well enough only in case of the concurrence of the estimations $\hat{\theta}_{n}$ with their true values $\theta_{n}$ $(n=1, \ldots, N)$ or if the estimations $\hat{\theta}_{n}$ differ from their true values by some constant value or linear addition along $n$ $(n=1, \ldots, N)$. In all other cases, the image (17) will be destroyed. Further we suggest an algorithm for the evaluation of the phase distortions $\hat{\theta}_{n}$.

\section{EVALUATION OF PHASE DISTORTIONS}

We used the criterion (7) in the algorithm for the evaluation of the phase distortions. The approach developed in Appendix A is valid only for the true functions $E(\vec{s})$ for which the expression (A.2) is in place. This means that the functions $E(\vec{s})$ have a constant complex phase multiplier over the whole scattering surface. Of course, (A.2) is not correct in a general case. However, for the objects of homogeneous material, (A.2) can be close to reality. In [1], the presented examples also use the approximation (A.2). We should note that the approximation (A.2) looks too strong, since normally the phase shift $\varphi(\vec{s})$ of each scatterer in the far zone is understood as its range phase shift. In the expression (12), the phase shift of the scatterer caused by its range is not included into $E(\vec{s})$, but it is incorporated into the phase multiplier $2 \vec{e}_{n}^{T} \cdot \vec{s} / c$, where $\vec{s}$ is a three-dimensional vector on the surface of the object. In other words, the phase shift of each scatterer due to its range is not a parameter to be estimated, but it is the parameter of the algorithm (17). The expression (17) is constructed by using all the possible vectors $\vec{s}$. Thus, we will use the criterion (7).

The complete exhaustive search across all the values of the phase distortions is impossible. Therefore, we have chosen an approximately optimal algorithm described below (formulas (24) and (25)). We mentioned above that the signals' ranges $\lambda$ (represented in time units (12)) lie within the interval $\left(\lambda_{\min }, \lambda_{\max }\right)$. We divide this interval into the range cells, each of a size equal to the range resolution. In terms of the time units, the resolution $\operatorname{del} \lambda$ is equal to $\operatorname{del} \lambda \approx 1.0 / \Delta \omega$, where $\Delta \omega$ is the signal frequency deviation. The number $M_{\lambda}$ of these range (time) cells is $M_{\lambda}=\left(\lambda_{\max }-\lambda_{\min }\right) / \operatorname{del} \lambda$. We will designate the number of each range cell as $m\left(1 \leq m \leq M_{\lambda}\right)$. The algorithm for the search of the unknown estimations $\hat{\theta}_{n}$ $(n=1, \ldots, N)$ comprises the following operations.

At the first stage, all the phases $\hat{\theta}_{n}(n=1, \ldots, N)$ are taken as those of the signals $Z_{n}(\lambda)(n=1, \ldots, N)$ in the first range cell with $m=1$. By using the estimations $\hat{\theta}_{n}$, we will construct the image by formulas (17) and (22) (in the whole frame) and calculate the functional (7)

$$
I_{1}=\int_{\Omega_{\vec{s}}} \hat{A}(\vec{s}) d^{2} \vec{s} .
$$

Then we will repeat the operations for the cell $m=2$. If the value of the criterion $I_{2}<I_{1}$, the estimations $\hat{\theta}_{n}$, obtained at the second step, look more preferable than the results of the first step. All these operations are repeated $M_{\lambda}$ times. Finally, the phases of $Z_{n}\left(\lambda_{\text {opt }}\right)(n=1, \ldots, N)$ at the range $\lambda_{\text {opt }}$ in the 
cell with number $m_{\mathrm{opt}}$, satisfying the criterion

$$
I_{\mathrm{opt}}=\min \left(I_{m}\right), \quad 1 \leq m \leq M_{\lambda},
$$

are taken as the final estimations $\hat{\theta}_{n}(n=1, \ldots, N)$ and are substituted in the final image estimations $\hat{E}(\vec{s})$ (see $(17)$ ). The estimations of the phase distortions obtained by this process are approximate. However, the search is performed in a 1-dimensional space and can be very fast. The results of the reconstruction are shown in Section 6.

Figure 1 shows the diagram of the algorithm (24) and (25) for finding the best compensating phases.

\section{RESULTS OF DIGITAL SIMULATION}

Figures 2, 3, 4, 5, 6, 7, 8, 9, 10, and 11 present the results of the digital simulation of the radar images. The threedimensional object is a Buratino doll with a dog on a chain. The object consists of spheres, cylinders, and prisms and it was simulated by using "OpenGL" package of 3D graphics [39].

Figure 2 shows the true object in the image plane inside the frame of $256 \times 256$ pixels. The vertical axis is the range (the radiation is directed from the bottom to the top) and the horizontal axis is the component of the object velocity, orthogonal to the middle vector of viewing. The parameters of the signals were taken as the typical ones for the American station LRIR (Long Range Imaging Radar), well known as Haystack [38], where the wavelength is $3.0 \mathrm{~cm}$ and the signal band is $1.0 \mathrm{GHz}$ for each radiated pulse.

Figure 3 presents the radar image of the object. The range resolution is $0.3 \mathrm{~m}$.

Figure 4 presents the similar image for the bandwidth of $300 \mathrm{MHz}$ for each pulse. The range resolution equals to $1.0 \mathrm{~m}$.

The following parameters are the same for all figures: the number of pixels is $256 \times 256$; the coherent processing of 256 pulses has been fulfilled. The scale division of the frame is $0.3 \mathrm{~m}$. A circular Keplerian orbit with the range of $450 \mathrm{~km}$ to the center of the masses of the object has been modeled. The radiation rate was 0.005 seconds.

Figures 3 and 4 present the images reconstructed in the absence of any additive noise in the signals.

Figure 5 presents the image with the maximum disturbance of coherence in the signals. These signals are obtained by adding a random value to the phase of each received signal, and this random value is uniformly distributed within $(-\pi, \pi)$. In Figure 5 the image is also reconstructed in the absence of any additive noise. The bandwidth is $1000 \mathrm{MHz}$. The image, in fact, disappeared, and the only remaining possibility is to make estimations of its sizes along the range axis.

Figure 6 presents the image for the case when the phase distortions compensation algorithm is applied. We can easily see that the object is restored with certain loss of the resolution. Also the image may have some shift along the velocity coordinate, because the algorithm reconstructs the un-

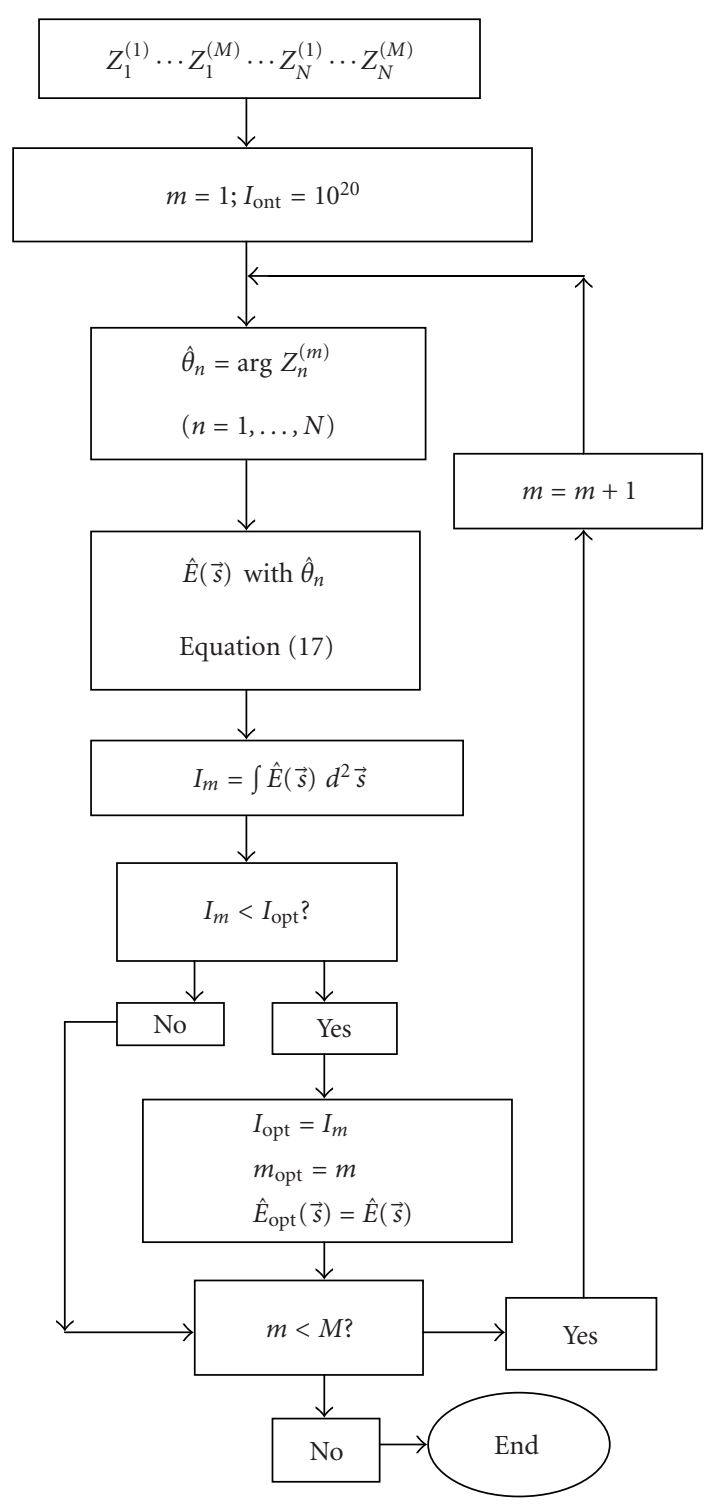

Figure 1: Diagram of the algorithm for finding the best compensating phases.

known phases $\theta_{n}(n=1, \ldots, N)$ with some possible constant or linear addition along $n$. This leads to the restoration of the image with the shift of the angle position in the frame.

In Figure 7, the image is reconstructed when the signal/additive noise ratio is equal to 2.0 in each element of the range resolution. The bandwidth is $1000 \mathrm{MHz}$. The phase distortions have the same statistical characteristics as those in Figure 5 . We can see that the image is worse than in Figure 5. Because of the significant additive noise, the image has disappeared even along the range coordinate.

Figure 8 presents the image after applying the compensating algorithm. For comparison, Figure 9 represents the image with the same parameters as in Figure 7 but without any phase distortions. Figures 8 and 9 make it clear that it is useful to apply the algorithm compensating the phase 


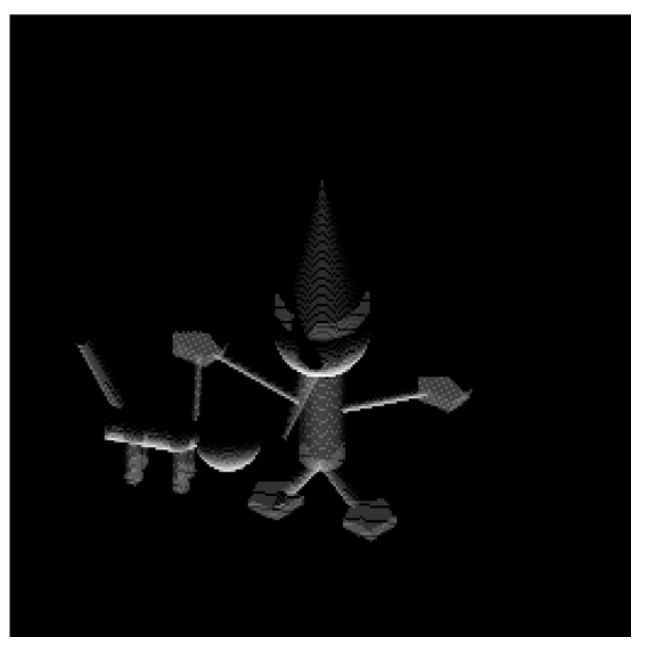

Figure 2: The true image "Buratino doll with a dog on a chain."

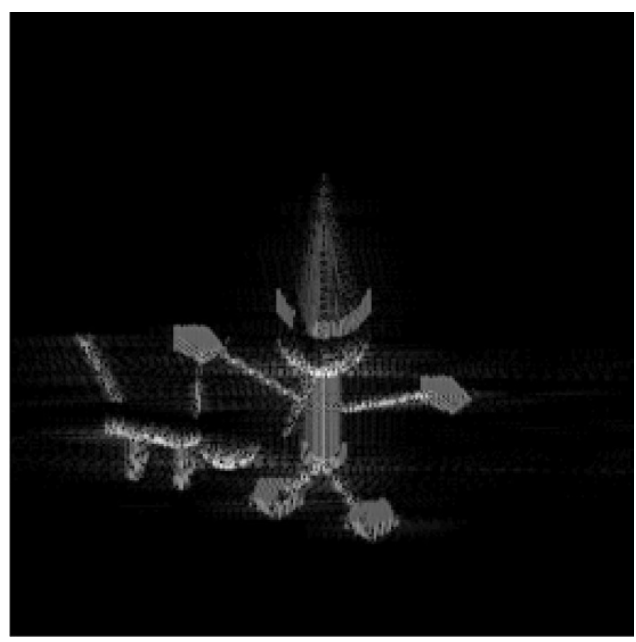

FIGURE 3: Radar image with bandwidth $1 \mathrm{GHz}$.

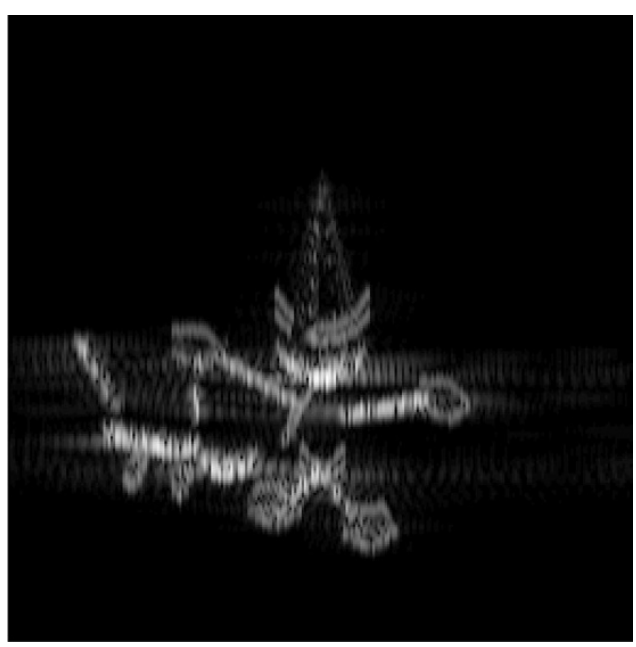

FIGURE 4: Radar image with bandwidth $300 \mathrm{MHz}$.

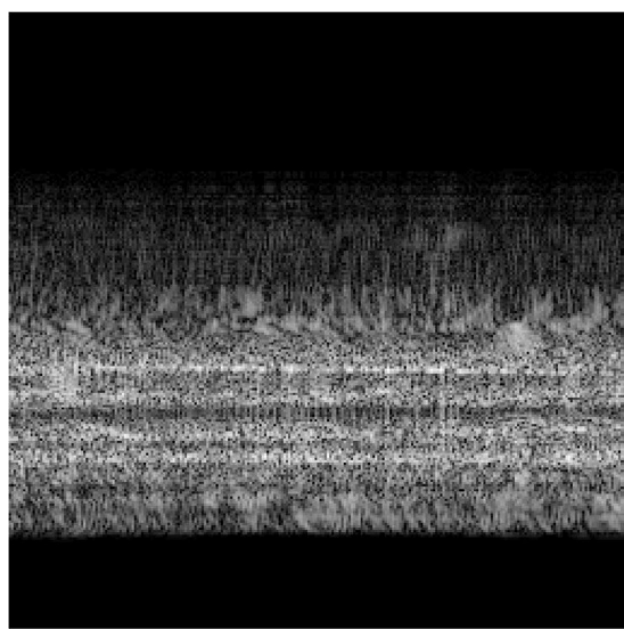

FIGURE 5: Radar image with phase distortions and bandwidth $1 \mathrm{GHz}$ without additive noises.

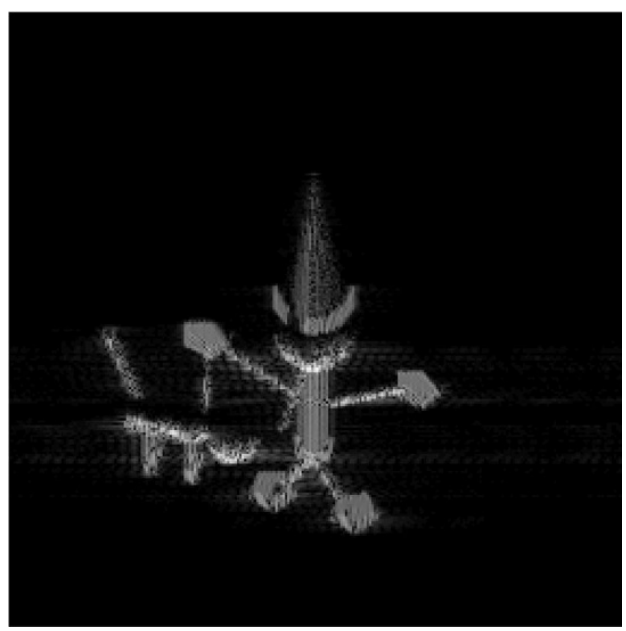

FIGURE 6: Image after the compensation phase distortions.

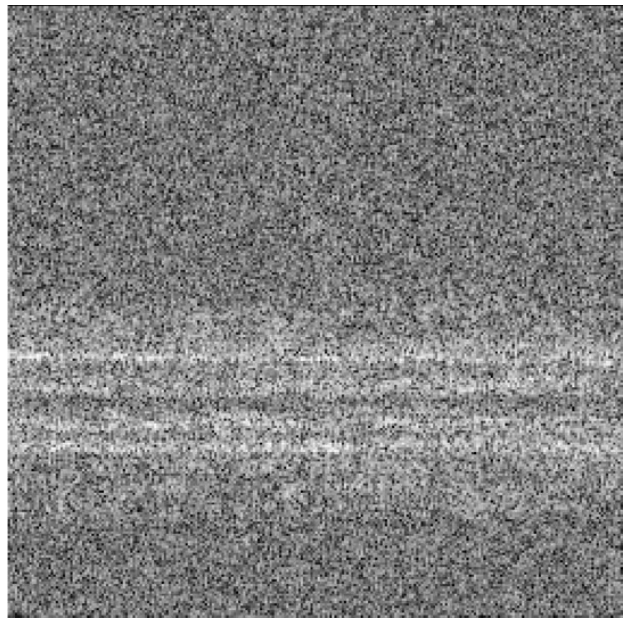

Figure 7: Radar image with phase distortions, bandwidth $1 \mathrm{GHz}$, and signal $/$ noise $=2$. 


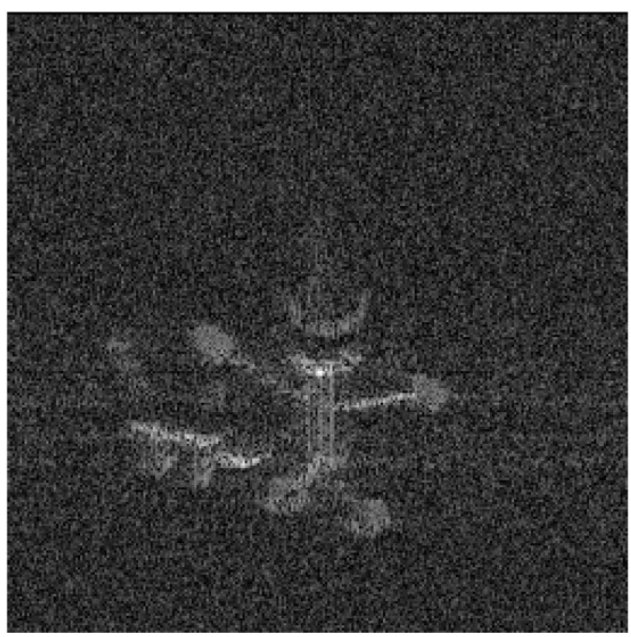

FIGURE 8: Image after the compensation phase distortions.

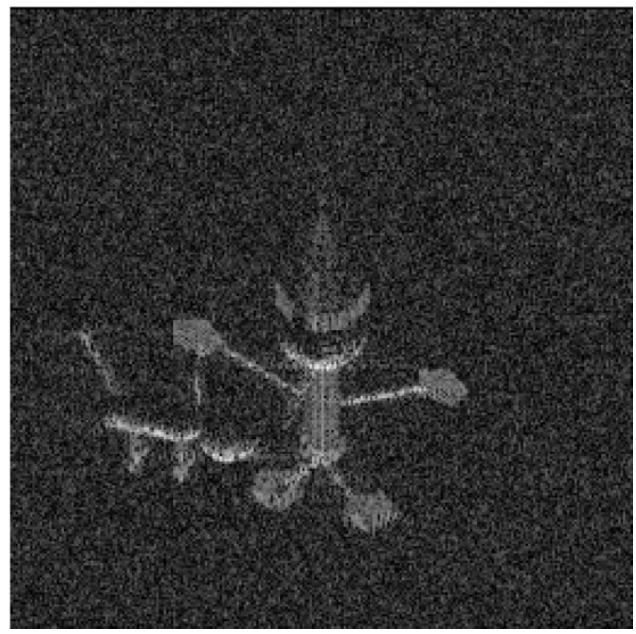

FIGURE 9: Radar image without phase distortions, bandwidth $1 \mathrm{GHz}$ and signal/noise $=2$.

distortions even for small signal/additive noise ratios when the phase distortions are present. The algorithm allows to recover the image with the same quality as that one obtained in the absence of any phase distortions. Figure 10 presents the image with the signal/noise ratio equal to 2.0 at the bandwidth of $300 \mathrm{MHz}$ with phase distortions, uniformly distributed within $(-\pi, \pi)$. Figure 11 presents the result of compensation. It is clear from Figures 8 and 11 that the higher the resolution range, the better the result of compensation for the same level of the additive noise.

\section{DISCUSSION}

The criterion was suggested for choosing the best image out of all the possibly obtained ones as a result of applying the inverse Fourier transform and using a compensating expo-

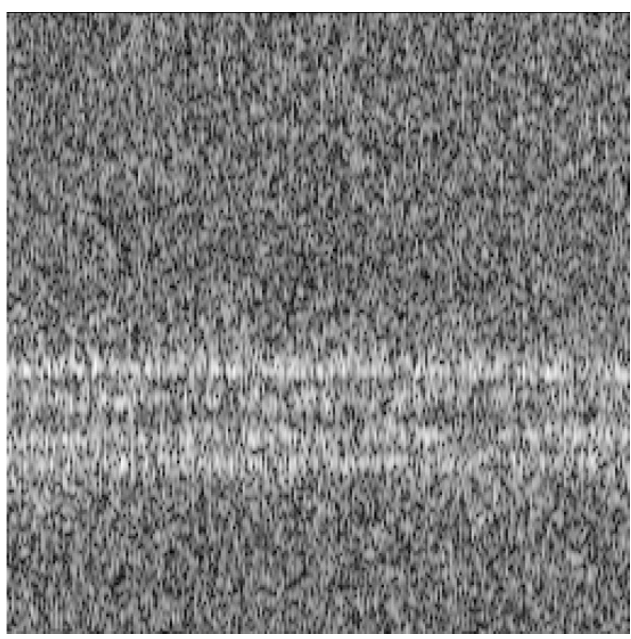

FIgURE 10: Radar image with phase distortions, bandwidth $300 \mathrm{MHz}$, and signal $/$ noise $=2$.

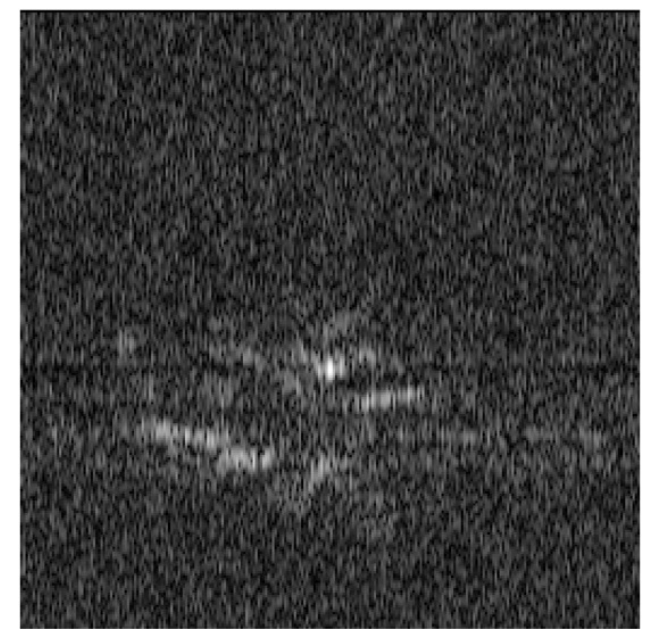

FIGURE 11: Image after the compensation phase distortions.

nential phase multiplier, when this Fourier transform contains some phase distortions. This criterion was applied to the task of the radar image recovery for the case of the signal phase distortions. The quality of the radar images was analyzed with regard to the parameters of the signals and the phase distortions. The digital simulation demonstrates that it is possible to restore the lost coherence in the received radar signals. So, the evaluation algorithm for the phase distortions was synthesized. The reliable performance of this algorithm requires a signal/noise ratio in each element of the range resolution of more than 2 .

The solution to the task by the method of "vector space projections" $[23,24,25,26]$ could possibly give more effective results of the compensation than the algorithm presented in Figure 1, but this is a particular and big task and is to be considered elsewhere. 


\section{APPENDICES}

\section{A. PROOF OF THE CRITERION FOR CHOOSING THE BEST IMAGE}

We assume that we have a true two-dimensional complex image

$$
E(\vec{s})=A(\vec{s}) \exp (j \varphi(\vec{s})),
$$

where $A(\vec{s})$ are the amplitudes of image and $\varphi(\vec{s})$ are the phases of the image. We will limit the study to the images for which

$$
\varphi(\vec{s})=\varphi_{0}=\text { const. }
$$

Further on, we will assume that the function $E(\vec{s})$ is unknown and we will have to construct its estimation. We denote this estimation as $\hat{E}(\vec{s})$. We can only obtain this estimation as a result of the two-dimensional Fourier transform of the function $\hat{F}(\vec{R})$. This can be considered the field generated by the object at point $\vec{R}$ of the receiving aperture in the far zone. The function $\hat{F}(\vec{R})$ is the distorted field of the true object

$$
\begin{aligned}
\hat{F}(\vec{R}) & =\exp (j \theta(\vec{R})) \int_{\Omega_{\vec{s}}} E(\vec{s}) \exp \left(j k_{0} \vec{R} \vec{s}\right) d^{2} \vec{s} \\
& =\exp (j \theta(\vec{R})) F(\vec{R}),
\end{aligned}
$$

where the true spectrum of the object is

$$
F(\vec{R})=\int_{\Omega_{\vec{s}}} E(\vec{s}) \exp \left(j k_{0} \vec{R} \vec{s}\right) d^{2} \vec{s} .
$$

In (A.3), $\theta(\vec{R})$ are the phase distortions and

$$
k_{0}=\frac{2 \pi}{\Lambda \rho}
$$

where $\Lambda$ is the wavelength of the radiation, $\rho$ is the range to the object $\Omega_{\vec{s}}$ is the area a priori occupied by the object.

The expression (A.3) means that the spectrum of the object is distorted in the phase, but not in the amplitude.

We will assume that the estimation of the object $\hat{E}(\vec{s})$ for point $\vec{s}$ is calculated as the inverse Fourier transform of the distorted spectrum $\hat{F}(\vec{R})$ with a compensating multiplier $\exp (-j \hat{\theta}(\vec{R}))$, in which

$$
\hat{E}(\vec{s})=\frac{1}{(2 \pi)^{2}} \int_{\Omega_{\vec{R}}} \hat{F}(\vec{R}) \exp \left(-j k_{0} \vec{R} \vec{s}\right) \exp (-j \hat{\theta}(\vec{R})) d^{2} \vec{R} .
$$

Here, $\hat{\theta}(\vec{R})$ is an arbitrary chosen estimation of the phase distortions and $\Omega_{\vec{R}}$ is the area of the receiving aperture.

Taking into account condition (A.2), we will build up the mere estimation $\hat{A}(\vec{s})$ of the amplitudes $A(\vec{s})$. It is natural to take this estimate as a modulus of the complex function (A.6):

$$
\begin{aligned}
\hat{A}(\vec{s}) & =|\hat{E}(\vec{s})| \\
& =\frac{1}{(2 \pi)^{2}}\left|\int_{\Omega_{\vec{R}}} \hat{F}(\vec{R}) \exp \left(-j k_{0} \vec{R} \vec{s}\right) \exp (-j \hat{\theta}(\vec{R})) d^{2} \vec{R}\right| .
\end{aligned}
$$

Further on, we will show that any arbitrary estimation of the distortions $\hat{\theta}(\vec{R})$ (with any true function $\theta(\vec{R})$ ) gives the estimation $\hat{A}(\vec{s})$ that satisfies the condition

$$
\int_{\Omega_{\vec{s}}} \hat{A}(\vec{s}) d^{2} \vec{s} \geq \int_{\Omega_{\vec{s}}} A(\vec{s}) d^{2} \vec{s}
$$

Here, $A(\vec{s})$ is the true image as mentioned above.

First of all, we evaluate the integral

$$
J_{0}=\left|\int_{\Omega_{\vec{s}}} \hat{E}(\vec{s}) d^{2} \vec{s}\right|
$$

By inserting (A.6) into (A.9) and changing the order of the integration, we will obtain

$$
\begin{aligned}
J_{0}= & \left|\int_{\Omega_{\vec{s}}} \hat{E}(\vec{s}) d^{2} \vec{s}\right| \\
= & \mid \frac{1}{(2 \pi)^{2}} \int_{\Omega_{\vec{R}}} F(\vec{R}) \exp (j(\theta(\vec{R})-\hat{\theta}(\vec{R}))) d^{2} \vec{R} \\
& \cdot \int_{\Omega_{\vec{s}}} \exp \left(-j k_{0} \vec{R} \vec{s}\right) d^{2} \vec{s} \mid .
\end{aligned}
$$

If the area $\Omega_{\vec{s}}$ is large enough (in other words, it contains a large number of the spatial resolution elements), we can rewrite (A.10) as

$$
J_{0}=\left|\int_{\Omega_{\vec{R}}} F(\vec{R}) \exp (j(\theta(\vec{R})-\hat{\theta}(\vec{R}))) \delta^{(2)}(\vec{R}) d^{2} \vec{R}\right|
$$

Here, $\delta^{(2)}(\vec{R})$ is a two-dimensional delta function.

The equation

$$
J_{0}=|F(0) \exp (j(\theta(0)-\hat{\theta}(0)))|=|F(0)|
$$

follows from (A.11).

By inserting (A.4) into (A.12), we finally have

$$
J_{0}=\left|\int_{\Omega_{\vec{\xi}}} \hat{E}(\vec{s}) d^{2} \vec{s}\right|=\left|\int_{\Omega_{\vec{\xi}}} E(\vec{s}) d^{2} \vec{s}\right| .
$$

For any complex function $\hat{E}(\vec{s})$, the following relationship is always valid:

$$
\int_{\Omega_{\vec{s}}} \hat{A}(\vec{s}) d^{2} \vec{s}=\int_{\Omega_{\vec{s}}}|\hat{E}(\vec{s})| d^{2} \vec{s} \geq J_{0}=\left|\int_{\Omega_{\vec{s}}} \hat{E}(\vec{s}) d^{2} \vec{s}\right| .
$$


It is clear from (A.1) and (A.2) that the true image satisfies

$$
E(\vec{s})=A(\vec{s}) \exp \left(j \varphi_{0}\right) .
$$

Now, (A.8) follows from (A.13), (A.14), and (A.15).

\section{B. SOLUTION TO THE MAXIMUM LIKELIHOOD EQUATION}

The calculation of $\delta \operatorname{Ln} P / \delta \operatorname{Re} E(\vec{s})$ yields

$$
\frac{\delta \operatorname{Ln} P}{\delta \operatorname{Re} E(\vec{s})}=\frac{2 \operatorname{Re}}{\sigma_{\text {noise }}^{2}}\left\{\sum_{n=1}^{N} \int_{\lambda_{\min }}^{\lambda_{\max }}\left(Z_{n}(\lambda)-\bar{Z}_{n}(\lambda)\right) \frac{\delta \bar{Z}_{n}^{*}(\lambda)}{\delta \operatorname{Re} E(\vec{s})} d \lambda\right\} .
$$

The functional derivative $\delta \bar{Z}_{n}^{*}(\lambda) / \delta \operatorname{Re} E(\vec{s})$ is, from (12),

$$
\begin{aligned}
\frac{\delta \bar{Z}_{n}^{*}(\lambda)}{\delta \operatorname{Re} E(\vec{s})}=\int_{S_{\text {LIGHT }}} & \frac{\delta \operatorname{Re} E\left(\vec{s}_{1}\right)}{\delta \operatorname{Re} E(\vec{s})} \exp \left(j \omega \tau_{n} \frac{\left(\vec{s}_{1}\right)}{c}\right) \\
& \cdot C_{0}\left(\lambda-\frac{\tau_{n}\left(\vec{s}_{1}\right)}{c}\right) \cdot d^{2} \vec{s}_{1} \exp \left(-j \theta_{n}\right) .
\end{aligned}
$$

Here, $\tau_{n}(\vec{s})$ is the designation of (18).

In (B.2), $\delta \operatorname{Re} E\left(\vec{s}_{1}\right) / \delta \operatorname{Re} E(\vec{s})$ is the functional derivative of the function with respect to itself and is equal to

$$
\frac{\delta \operatorname{Re} E\left(\vec{s}_{1}\right)}{\delta \operatorname{Re} E(\vec{s})}=\delta^{2}\left(\vec{s}_{1}-\vec{s}\right)
$$

$(\vec{s}$ is an argument of $\operatorname{Re} E(\vec{s}))$.

After inserting (B.3) into (B.2) and (B.2) into (B.1), we obtain

$$
\begin{array}{r}
\frac{\delta \operatorname{Ln} P}{\delta \operatorname{Re} E(\vec{s})}=\frac{2 \operatorname{Re}}{\sigma_{\text {noise }}^{2}}\left\{\sum_{n=1}^{N} \int_{\lambda_{\min }}^{\lambda_{\max }} Z_{n}(\lambda) C_{0}^{*}\left(\lambda-\tau_{n}(\vec{s})\right) d \lambda\right. \\
\left.\quad \times \exp \left(j\left(\theta_{n}-\omega \tau_{n}(\vec{s})\right)\right)\right\}-\Phi,
\end{array}
$$

where $\Phi$ is the functional

$$
\begin{aligned}
& \Phi= \frac{2 \operatorname{Re}}{\sigma_{\text {noise }}^{2}} \\
& \times\left\{\sum_{n=1}^{N} \int_{\lambda_{\min }}^{\lambda_{\max }} \int_{S_{\text {LIGHT }}} E^{*}\left(\vec{s}_{1}\right) \exp \left(j \omega \tau_{n}\left(\vec{s}_{1}\right)\right) C_{0}\left(\lambda-\tau_{n}\left(\vec{s}_{1}\right)\right)\right. \\
& \cdot C_{0}^{*}\left(\lambda-\tau_{n}(\vec{s})\right) d^{2} \vec{s}_{1} d \lambda \\
& \\
&\left.\quad \times \exp \left(-j \omega \tau_{n}(\vec{s})\right)\right\} .
\end{aligned}
$$

The calculation of the internal integral over $\lambda$ in (B.5) leads to

$$
\begin{gathered}
\Phi=\frac{2 \operatorname{Re}}{\sigma_{\text {noise }}^{2}}\left\{\sum_{n=1}^{N} \int_{S_{\text {LIGHT }}} E^{*}\left(\vec{s}_{1}\right) \exp \left(j \omega \tau_{n}\left(\vec{s}_{1}\right)\right) \delta\left(\tau_{n}\left(\vec{s}_{1}\right)-\tau_{n}(\vec{s})\right)\right. \\
\left.\times \exp \left(-j \omega \tau_{n}(\vec{s})\right) \cdot d^{2} \vec{s}_{1}\right\} .
\end{gathered}
$$
that is,

The assumption of a high signal resolution has been used,

$$
\int_{\lambda_{\min }}^{\lambda_{\max }} C_{0}\left(\lambda-\tau_{1}\right) C_{0}^{*}\left(\lambda-\tau_{2}\right) d \lambda \approx \delta\left(\tau_{1}-\tau_{2}\right) .
$$

After the integration along $\vec{s}_{1}$ in (B.6), we get

$$
\Phi=\frac{N 2 \operatorname{Re} E^{*}(\vec{s})}{\sigma_{\text {noise }}^{2}} .
$$

By inserting (B.8) into (B.4) and using (16), we obtain the estimate of the real part of $\hat{E}^{*}(\vec{s})$ :

$$
\operatorname{Re} \hat{E}^{*}(\vec{s})=\frac{\operatorname{Re}}{N}\left\{\sum_{n=1}^{N} Z_{n}\left(\tau_{n}(\vec{s})\right) \exp \left(j\left(\theta_{n}-\omega \tau_{n}(\vec{s})\right)\right)\right\} .
$$

The similar considerations will lead to the estimation of the imaginary part of $\hat{E}^{*}(\vec{s})$ :

$$
\operatorname{Im} \hat{E}^{*}(\vec{s})=-\frac{\operatorname{Im}}{N}\left\{\sum_{n=1}^{N} Z_{n}\left(\tau_{n}(\vec{s})\right) \exp \left(j\left(\theta_{n}-\omega \tau_{n}(\vec{s})\right)\right)\right\} .
$$

The estimations (B.9) and (B.10) are obtained under the assumption that the values $\theta_{n}$ are exactly known.

If $\theta_{n}(n=1, \ldots, N)$ are unknown, their estimations $\hat{\theta}_{n}$ $(n=1, \ldots, N)$ should be substituted into (B.9) and (B.10). The expressions (B.9) and (B.10) compose the operation (17).

\section{REFERENCES}

[1] Z. S. She, D. A. Gray, and R. E. Bogner, "Autofocus for inverse synthetic aperture radar (ISAR) imaging," Signal Processing, vol. 81, no. 2, pp. 275-291, 2001.

[2] M. J. Prickett and C. C. Chen, "Principles of inverse synthetic aperture radar (ISAR) imaging," in Proc. IEEE Eascon Record (EASCON '80), pp. 340-345, Arlington, Va, USA, September 1980.

[3] H. Wu, D. Grenier, G. Y. Delisle, and D. G. Fang, "Translational motion compensation in ISAR image processing," IEEE Trans. Image Processing, vol. 4, no. 11, pp. 1561-1571, 1995.

[4] B. Kang, H. Subbaram, and B. D. Steinberg, "Improved adaptive-beamforming target for self-calibrating a distorted phased array," IEEE Trans. Antennas and Propagation, vol. 38, no. 2, pp. 186-194, 1990.

[5] F. Berizzi and G. Corsini, "Autofocusing of inverse synthetic aperture radar images using contrast optimization," IEEE Trans. on Aerospace and Electronics Systems, vol. 32, no. 3, pp. 1185-1191, 1996. 
[6] Z. S. She and Z. D. Zhu, "Two improved methods of motion compensation for 1D cross-range ISAR imaging," in Proc. IEEE National Aerospace and Electronics Engineering Conference (NAECON '96), pp. 227-230, Dayton, Ohio, USA, May 1996.

[7] C. V. Jakowatz and D. E. Wahl, "Eigenvector method for maximum-likelihood estimation of phase errors in syntheticaperture-radar imagery," Journal of Optical Society of America $\{A\}$, vol. 10, no. 12, pp. 2539-2546, 1993.

[8] B. D. Steinberg, "Microwave imaging of aircraft," Proceedings of the IEEE, vol. 76, no. 12, pp. 1578-1592, 1988.

[9] E. Yadin, "SAR autofocusing viewed as adaptive beamforming on prominent scatterers," in Proc. IEEE National Radar Conference, pp. 138-143, Atlanta, Ga, USA, March 1994.

[10] D. B. Ivashov and Y. V. Zhulina, "Potentials of the radar image reconstruction algorithms," Radiotekhnika i Elektronika, vol. 41, pp. 1-22, 1996.

[11] D. B. Ivashov and Y. V. Zhulina, "Building of images in a radar systems with a single receiver point, wideband signal, synthesis of aperture in time and compensation of phase distortions in signal," Radiotechnika, , no. 3, pp. 81-88, 1996.

[12] G. Ayers and J. C. Dainty, "Iterative blind deconvolution method and its applications," Optical Letters, vol. 13, no. 7, pp. 547-549, 1988.

[13] R. G. Lane, "Blind deconvolution of speckle images," Journal of Optical Society of America $\{A\}$, vol. 9, no. 9, pp. 1508-1514, 1992.

[14] S. M. Jefferies and J. C. Christou, "Restoration of astronomical images by iterative blind deconvolution," Astrophysical Journal, vol. 415, pp. 862-874, October 1993.

[15] T. J. Schulz, "Multiframe blind deconvolution of astronomical images," Journal of Optical Society of America $\{A\}$, vol. 10, no. 5, pp. 1064-1073, 1993.

[16] E. Thiebaut and J. M. Conan, "Strict a priori constraints for maximum-likelihood blind deconvolution," Journal of Optical Society of America $\{A\}$, vol. 12, no. 3, pp. 485-492, 1995.

[17] Y. Yang, N. P. Galatsanos, and H. Stark, "Projection based blind deconvolution," Journal of Optical Society of America $\{A\}$, vol. 11, no. 9, pp. 2401-2409, 1994.

[18] N. F. Law and R. G. Lane, "Blind deconvolution using least square minimisation," Optics Communications, vol. 128, pp. 341-352, 1996.

[19] A. Kuriksha and Y. V. Zhulina, "Restoration distorted images in optical systems with Poisson fluctuations of the signal in a photoreceiver," Radiotekhnika i Elektronika, vol. 45, no. 3, pp. 313-319, 2000.

[20] S. C. Douglas, A. Cichocki, and S. Amari, "Self-whitening algorithms for adaptive equalization and deconvolution," IEEE Trans. Signal Processing, vol. 47, no. 4, pp. 1161-1165, 1999.

[21] R. J. Hanish and R. L. White, Eds., The Restoration of HST Images and Spectra-II, Proceedings of a Workshop Held at the Space Telescope Science Institute, Baltimore, Md, USA, 1993.

[22] F. Goudail, O. Ruch, and Ph. Refregier, "Deconvolution of several versions of a scene perturbed by different defocus blurs: influence of kernel diameters on restoration quality and on robustness to kernel estimation," Applied Optics-IP, vol. 39, no. 35, pp. 6602-6612, December 2000.

[23] H. Stark and Y. Yang, Vector Space Projections: A Numerical Approach to Signal and Image Processing, Neural Nets, and Optics, John Wiley \& Sons, NY, USA, 1998.

[24] K. C. Haddad, H. Stark, and N. P. Galatsanos, "Constrained FIR filter design by the method of vector space projections," IEEE Trans. on Circuits and Systems II: Analog and Digital Signal Processing, vol. 47, no. 8, pp. 714-725, 2000.
[25] K. C. Haddad, H. Stark, and N. P. Galatsanos, "Design of digital linear-phase FIR crossover systems for loudspeakers by the method of vector space projections," IEEE Trans. Signal Processing, vol. 47, no. 11, pp. 3058-3066, 1999.

[26] Y. Yang and H. Stark, "Design of self-healing arrays using vector-space projections," IEEE Trans. Antennas and Propagation, vol. 49, no. 4, pp. 526-534, 2001.

[27] B. D. Steinberg, "Microwave imaging of aircraft," Proceedings of the IEEE, vol. 76, no. 12, pp. 1578-1592, 1988.

[28] D. R. Wehner, High Resolution Radar, Artech House, Norwood, Mass, USA, 1995.

[29] D. A. Ausherman, A. Kozma, J. L. Walker, H. M. Jones, and E. C. Poggio, "Developments in radar imaging," IEEE Trans. on Aerospace and Electronics Systems, vol. 20, no. 4, pp. 363400, 1984.

[30] C. C. Chen and H. C. Andrews, "Target-motion-induced radar imaging," IEEE Trans. on Aerospace and Electronics Systems, vol. 16, no. 1, pp. 2-14, 1980.

[31] C. Elachi, T. Bicknell, R. L. Jordan, and C. Wu, "Spaceborne synthetic-aperture imaging radars: applications, techniques and technology," Proceedings of the IEEE, vol. 70, no. 10, pp. 1174-1209, 1982.

[32] S. L. Borison, S. B. Bowling, and K. M. Cuomo, "Superresolution methods for wideband radar," The Lincoln Laboratory Journal, vol. 5, no. 3, pp. 441-461, 1992.

[33] T. P. Wallace, "An all-neighbor classification rule based on correlated distance combination," Tech. Rep. 1030, MIT Lincoln Laboratory, Lexington, Mass, USA, November 1996.

[34] G. P. Banner, "Deep space surveillance-overview and radar tracking," in Proc. 3rd US/Russia Space Surveillance Workshop, Washington, DC, USA, October 1998.

[35] D. Mehrholz and K. Magura, "Radar tracking and observation of noncooperative space objects by reentry of Salyut7/Kosmos-1686," in Proc. International Workshop ESOC on Salyut-7/Kosmos-1686 Reentry, number ESA SP-345, pp. 1-8, Darmstadt, Germany, April 1991.

[36] D. Mehrholz, "Potentials and limits of space object observations and data analyses using radar techniques," in Proc. 1st European Conference on Space Debris, number ESA-SD-01, Darmstadt, Germany, April 1993.

[37] E. C. Pearce, "Satellite photometry with the GEODSS and MOSS systems," in Proc. 4th US/Russia Space Surveillance Workshop, October 2000.

[38] C. V. Solodyna and G. P. Banner, "Narrowband and wideband radar signatures in support of the space catalog," in Proc. 4th US/Russia Space Surveillance Workshop, October 2000.

[39] J. D. Foley, A. van Dam, S. K. Feiner, and J. F. Hughes, Computer Graphics: Principles and Practice, Addison-Wesley, NY, USA, 1991.

Yulia V. Zhulina was born in Igarka, Russia, in 1940. She received her B.S. degree in radio engineering from Moscow Physical and Engineering Institute, Moscow, Russia, in 1963. She received her Ph.D. degree in radar engineering from Moscow Physical and Engineering Institute, Moscow, Russia, in 1968. In 1963, she joined the Radar Engineering Department at "Vympel," where she is currently a Senior Scientist Researcher. She is a Coauthor of the book Detecting Moving Objects (Moscow, Sovetskoye Radio) in 1980. Her research interests are in image recovery, medical, optical, radar imaging, methods of the "blind deconvolution," recognition with the optical images, and applied mathematical and statistical methods.

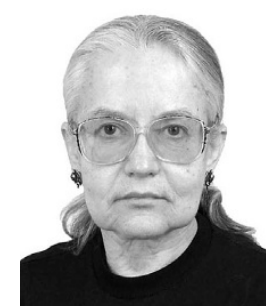

